\title{
Analysis of Subcycle Electro-Optic Sampling Without Background
}

\author{
Cornelius Beckh $^{1}$ (D) Philipp Sulzer ${ }^{1}$ (D) - Niklas Fritzsche ${ }^{1} \cdot$ Claudius Riek $^{1}$. \\ Alfred Leitenstorfer ${ }^{1}$ (D)
}

Received: 21 January 2021 /Accepted: 23 March 2021/Published online: 7 April 2021

(C) The Author(s) 2021

\begin{abstract}
We explore background-free options to detect mid-infrared (MIR) electric transients. The MIR field and a near-infrared probe interact via sum- (SFG) and difference-frequency generation (DFG) in an electro-optic crystal. An intuitive picture based on a phasor representation and rigorous numerical calculations are used for analysis. It turns out that separating photons generated either by SFG or DFG from the local oscillator via spectral filtering leads to a signal purely proportional the MIR intensity envelope. Backgroundfree phase information may be extracted in a spectral window containing both SFG and DFG components and blocking the local oscillator background based on its orthogonal polarization. This variant leads to signal proportional to the square of the MIR field amplitude. It is limited by the finite efficiency of polarization filtering. The Hilbert transform as a conjugate variable to the electric field in the time domain turns out to play a fundamental role for the context discussed in this paper.
\end{abstract}

Keywords THz detection · Electro-optic sampling - Background-free · Hilbert transform · Timedomain quantum optics

\section{Introduction}

Recent progress of electro-optic sampling (EOS) in the terahertz (THz) and mid-infrared (MIR) frequency ranges has opened up direct access to the vacuum fluctuations of the electric field $[1,2]$, and routes towards quantum optics operating with subcycle resolution in the time domain are currently being developed [3-5]. While cryogenic cooling is required in the $\mathrm{THz}$

\section{Cornelius Beckh}

cornelius.beckh@googlemail.com

Alfred Leitenstorfer

alfred.leitenstorfer@uni-konstanz.de

1 Department of Physics and Center for Applied Photonics, University of Konstanz,

D-78457 Konstanz, Germany 
range to freeze out thermal fluctuations of the field, the quantum limit is reached already at room temperature in the MIR. Nevertheless, also the concepts for detecting electric field amplitudes with few-femtosecond resolution [6,7] include an element of homodyne amplification where a new electric field generated by mixing with the longwave electric field is generated with a polarization direction perpendicular to the near-infrared (NIR) probe [8]. An ellipsometric analysis of the relative optical phase of this polarization state gives direct access to either the electric field or its conjugate variable in the time domain, namely, the Hilbert transform [9]. In these schemes, technical excess noise of the pulse train used for probing may be suppressed by a balanced detector, but the shot noise of the flux of coherent NIR photons remains as the limiting factor for quantum detection. In this contribution, we analyze potential options for ultrafast detection of electric fields which are not suffering from this restriction.

As a theoretical model, we consider the frequency-domain description of electro-optic sampling where the new field consists of photons created by difference- (DFG) and sumfrequency generation (SFG) between the MIR transient and the NIR probe [8]. Any isolated detection of the newly generated field avoids the shot-noise limitation by the probe.

In a first example, either SFG or DFG contributes to the signal. This option comes with the advantage that the new field may be spectrally separated from the probe. Consequently, spectral filtering is applicable and might allow direct characterization of the $\mathrm{THz}$ input by detecting sidebands. In case of a narrowband probe, only spectral information about the $\mathrm{THz}$ pulse may be gained [10]. Analyzing the temporal shape of the sideband by frequencyresolved optical gating (FROG) [11] also gives access to the $\mathrm{THz}$ envelope. Note that any phase information of the $\mathrm{THz}$ field cannot be extracted from those previous experiments because the duration of the probe pulse lasts several nanoseconds, i.e., much longer as compared to the oscillation cycle of the $\mathrm{THz}$ field.

In this work, the time resolution of sub-10-fs probe pulses allows us to study the temporal evolution of the spectral sidebands. Interestingly, we are observing a signal proportional to the intensity envelope of a MIR transient. The phase information vanishes which means that even in the zero crossings of the MIR transient, DFG and SFG photons are generated with the same amplitude as in the maxima of the electric field. The physics of this extreme case of a wavemixing process is studied where one of the interacting fields consists of a wave packet shorter than the oscillation cycle of the other input. Note that the principles we describe are valid irrespective of the frequencies under study. Consequently, the terms (multi-)THz and MIR are used alternately to denote the radiation that is examined.

In the second case, DFG and SFG are allowed to interfere and contribute equally to the signal. Here, contributions from the Hilbert transform of the field may be eliminated, and information about the phase of the THz field is retrieved except for its sign. The fact that full interference between SFG and DFG is possible only in the center of the probe spectrum excludes the option of spectral filtering. Suppression of the background has to rely on polarization filtering only. At high electric $\mathrm{THz}$ fields, a significant background might be tolerable owing to the large nonlinear phase shifts $[12,13]$. In this context, the carrier-envelope phase can be reconstructed by analyzing the interference pattern in a FROG setup [14]. Alternatively, the third-order nonlinearity may be exploited to detect an interference of DFG and SFG photons in a spectral window without background $[15,16]$. The question remains how far such concepts lead in characterizing extremely small $\mathrm{THz}$ amplitudes.

In this paper, we investigate the different options of background-free EOS, compare them with the standard configuration exploiting ellipsometry and homodyning, and discuss their potential for subcycle quantum electro-dynamics. Section 2 introduces the laser setup and the 
experimental implementations to detect a background-free EOS signal. In Section 3, an intuitive picture based on a monochromatic $\mathrm{THz}$ input and a phasor representation of the new fields illustrates the general features of the signal for both cases mentioned above. In Section 4, we study the full time evolution with numerical simulations based on the frequencydomain description by Gallot and Grischkowsky [8]. Finally, experimental results on background-free EOS are presented and discussed in Section 5. Section 6 summarizes the results and their consequences for time-domain quantum optics.

\section{Experimental Setup}

All measurements are based on a femtosecond erbium:fiber front end operating at a repetition rate of $40 \mathrm{MHz}$. It provides three amplified pulse trains, as depicted by black horizontal lines in Fig. 1. In the first branch, an electro-optic modulator (EOM) reduces the repetition rate to $10 \mathrm{MHz}$ before a highly nonlinear fiber (HNF) shifts the center wavelength to $1.03 \mu \mathrm{m}$ (corresponding to a frequency of $291 \mathrm{THz}$ ) and seeds a highpower ytterbium fiber amplifier (Yb:amp). HNF assemblies generate ultrabroadband spectra which support pulses with durations shorter than $10 \mathrm{fs}$ in the second and third branch. The first sub-10-fs pulse serves as a seed for an optical parametric amplifier (OPA) that is pumped with the second harmonic of the output of the Yb:fiber amplifier. The OPA provides up to $200 \mathrm{~nJ}$ pulse energy with pulse durations of $8.3 \mathrm{fs}[17,18]$. In this work, only $20 \mathrm{~nJ}$ is employed for optical rectification (OR) because of the damage threshold of the generation crystal (GX). A 16- $\mu$ m-thick gallium selenide (GaSe) crystal emits broadband MIR transients with frequency components up to $70 \mathrm{THz}$. A $500-\mu \mathrm{m}-$ thick germanium wafer inserted under Brewster's angle spatiotemporally superimposes the multi-THz radiation with the second sub-10-fs pulse serving as a probe. The repetition rate of the probe pulse is reduced to $20 \mathrm{MHz}$ with another EOM to twice the rate of the MIR pulse train. Its pulse duration is $7.7 \mathrm{fs}$, and the spectrum is centered at a frequency of $280 \mathrm{THz}$. The probe pulse energy of $250 \mathrm{pJ}$ is small compared to the pump. The linear extinction ratio of the probe polarization is better than $10^{-6}$ after reflection at the germanium wafer. The multi-THz transient and the NIR probe pulse are focused into a 12.2- $\mu$ m-thick GaSe electro-optic crystal (EOX) with an off-axis parabolic mirror. The relative timing is controlled by a variable optical delay line (VD). To allow optimal spectral separation of up- or down-converted photons, the spectrum of the probe is shaped either by edge filters (EF) before the Ge wafer or with razor blades located in front of the end mirror of a prism sequence. The compressor stage also compensates for the additional dispersion introduced by the edge filters. An increase of the pulse duration from $7.7 \mathrm{fs}$ up to a maximum of $10.5 \mathrm{fs}$ is monitored by frequencyresolved optical gating (FROG).

After collimation with an achromatic lens, the probe pulse can enter into two different detection setups A or B (see Fig. 1). In setup A, the electric field amplitude and phase of the MIR pulse may be determined by analyzing the change of ellipticity of the probe polarization. This standard way of EOS exploits a quarter-wave plate (QWP), a Wollaston prism (WP), and a balanced photodetector (BPD). In setup $\mathrm{B}$, the original probe pulse is suppressed by spectral band pass (BP) filters and a linear polarizer (Pol.).

The polarization states of the electric fields and the filtering sequence in detection scheme $\mathrm{B}$ are illustrated in the inset of Fig. 1. The probe field $\mathrm{E}_{\mathrm{p}}$ and the field of the multi-THz transient 


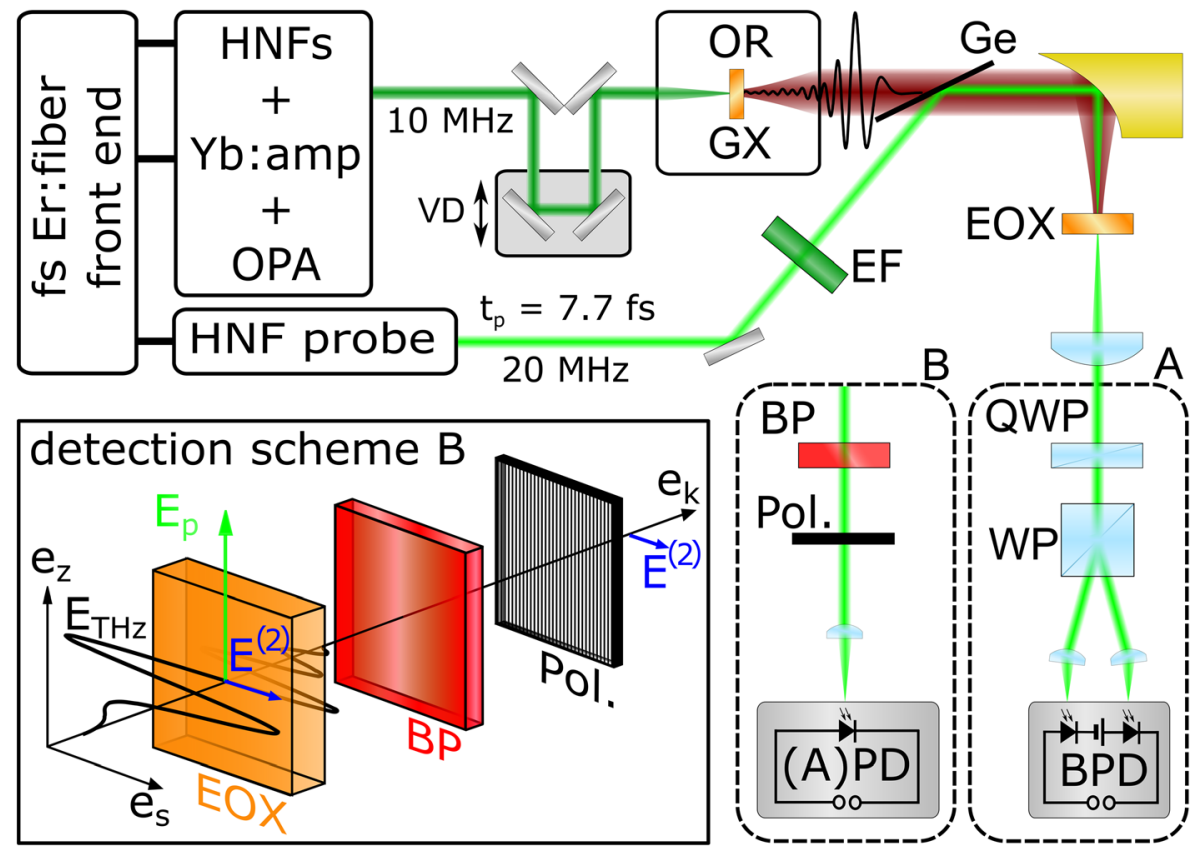

Fig. 1 Sketch of the experimental setup. The femtosecond laser source and THz generation are depicted on top, followed either by detection scheme A or B in the lower right corner. The polarization directions of the input field $\mathrm{E}_{\mathrm{THz}}$, the probe field $\mathrm{E}_{\mathrm{p}}$ and the newly generated field $\mathrm{E}^{(2)}$ are indicated with respect to unit vectors $\mathrm{e}_{\mathrm{s}}$ and $\mathrm{e}_{\mathrm{z}}$ in the lower left box, together with the optical components following along propagation direction $\mathrm{e}_{\mathrm{k}}$ for the case of detection scheme B. HNF, highly nonlinear fiber; Yb:amp, Yb-doped fiber amplifier; OPA, optical parametric amplifier; VD, variable delay stage; OR, optical rectification; GX, generation crystal; Ge, germanium filter and beam combiner; EF, edge filter; EOX, electro-optic crystal; BP, bandpass filter; Pol., linear polarizer; (A)PD, (avalanche) photodiode; QWP, quarter-wave plate; WP, Wollaston prism; BPD, balanced photodetector

$\mathrm{E}_{\mathrm{THz}}$ are linearly polarized but perpendicular to each other, pointing in the direction of $\mathrm{e}_{\mathrm{z}}$ and $\mathrm{e}_{\mathrm{s}}$, respectively. The newly generated field $\mathrm{E}^{(2)}$ is linearly polarized along $\mathrm{e}_{\mathrm{s}}$ and thus perpendicular to the original probe, enabling separation by a polarizer. The photons of $\mathrm{E}^{(2)}$ are detected either with a silicon avalanche photodiode (Si APD) for frequencies above 300 $\mathrm{THz}$ (corresponding to a wavelength of $1 \mu \mathrm{m}$ ) or an indium gallium arsenide photodiode (InGaAs PD) at lower frequencies, respectively. For both detection schemes A and B, a radiofrequency lock-in amplifier demodulates the electric signal at the repetition rate of the multi$\mathrm{THz}$ pulse train of $10 \mathrm{MHz}$.

\section{Phasor Representation}

In this section, we derive the expected signals for both detection schemes based on an intuitive phasor picture [9]. The nonlinear mixing process in the EOX between a NIR probe pulse and a monochromatic MIR pulse is described by DFG and SFG. All fields are regarded as spectral amplitudes only. The inset of Fig. 1 defines their polarization properties and vector characteristics. New field components $\mathrm{E}_{\mathrm{SFG}}$ and $\mathrm{E}_{\mathrm{DFG}}$ are generated mixing the multi-THz field $\mathrm{E}_{\mathrm{THz}}$ and the probe field $E_{p}$ of angular frequency $\Omega$ and $\omega_{p}=\omega \pm \Omega$, respectively. $\omega$ is the frequency at which sum- and difference-frequency components are created: 


$$
\begin{gathered}
E_{S F G}\left(\omega, t_{D}\right) \propto i \chi^{(2)} E_{T H z}(\Omega) E_{p}(\omega-\Omega) e^{-i \Omega t_{D}} \\
E_{D F G}\left(\omega, t_{D}\right) \propto i \chi^{(2)} E_{T H z}^{*}(\Omega) E_{p}(\omega+\Omega) e^{i \Omega t_{D}}
\end{gathered}
$$

Here, $\chi^{(2)}$ denotes the relevant element of the nonlinear susceptibility tensor and $t_{\mathrm{D}}$ the relative time delay between the input fields. The new polarization state of the MIR field emerges as the sum of the broadband probe $E_{\mathrm{p}}(\omega \pm \Omega)$ and the new field $E^{(2)}(\omega)=\mathrm{E}_{\mathrm{SFG}}+\mathrm{E}_{\mathrm{DFG}}$ pointing along $\mathrm{e}_{\mathrm{z}}$ and $\mathrm{e}_{\mathrm{s}}$, respectively. All complex-valued factors in Eqs. (1) and (2) contribute to the phase. In this ideal scenario $\chi^{(2)}, E_{\mathrm{THz}}$ and $E_{\mathrm{p}}$ are real valued. Hence at zero delay time $t_{\mathrm{D}}=0$, only the complex unit $i$ adds to the relative phase between $E_{\mathrm{p}}$ and $E_{\mathrm{THz}}$ with a factor of $\pi / 2$. An elliptical polarization of the total NIR field results. The polarization state changes when the delay time $t_{\mathrm{D}}$ is varied. In the standard case of electro-optic sampling, the ellipticity is analyzed and enables a direct determination of the electric field $E_{\mathrm{THz}}$. Recently, it was demonstrated that the Hilbert transform of the electric field can be determined if either DFG or SFG is exploited exclusively and the linear rotation angle of the NIR polarization is measured with a half-wave plate (HWP) instead of studying ellipticity with a QWP [9].

We now expand the phasor description based on Eqs. (1) and (2) to investigate the behavior of background-free signals where no homodyning with the original NIR field occurs. The situation is sketched in Fig. 2a where the relevant fields are represented by phasors in the complex plane for both detection schemes $\mathrm{A}$ and $\mathrm{B}$. The probe field $E_{\mathrm{p}}$ is defined to point constantly along the real axis $\Re$. At $t_{\mathrm{D}}=0$, the generated fields $\mathrm{E}_{\mathrm{DFG}}$ and $\mathrm{E}_{\mathrm{SFG}}$ are parallel to the imaginary axis $\mathfrak{I}$ because of their $\pi / 2$ relative phase shift with respect to the original probe. Owing to the delay-dependent phase factor in Eqs. (1) and (2) which is provided by the $\mathrm{THz}$ field at frequency $\Omega$ (black dashed arrows in Fig. $2 \mathrm{a}$ ), $E_{\mathrm{SFG}}$ rotates clockwise and $E_{\mathrm{DFG}}$ rotates counterclockwise as $t_{\mathrm{D}}$ increases. The new fields are illustrated with blue arrows for a fixed delay time $t_{\mathrm{D}} \neq 0$. The differential photocurrent $\Delta \mathrm{I} / \mathrm{I}$ resulting from the balanced detector of scheme A and the absolute current output I of a single photodiode in scheme B are plotted versus $t_{\mathrm{D}}$ in Fig. $2 \mathrm{~b}$ and c, respectively. We start with detection scheme A, representing the standard ellipsometry scheme for EOS. Here, the QWP projects $E_{\mathrm{DFG}}+E_{\mathrm{SFG}}$ to the imaginary axis $\mathfrak{I}$, as indicated by the black arrows in Fig. 2. The differential photocurrent $\Delta \mathrm{I} / \mathrm{I}$ measured by subtracting the two output ports of the WP is proportional to this projection. Consequently, the total output signal $S_{\mathrm{QWP}}$ represents the electric field amplitude $E_{\mathrm{THz}}\left(t_{\mathrm{D}}\right)$, as exemplified by the black graph in Fig. $2 \mathrm{~b}$.

In detection scheme $\mathrm{B}$, the original probe field is suppressed and no homodyning occurs. In contrast to scheme A, this fact renders the phase relationship to the original probe field $E_{\mathrm{p}}$ irrelevant. Therefore, only the relative phase between $E_{\mathrm{DFG}}$ and $E_{\mathrm{SFG}}$ is to be considered. Two limiting cases may be distinguished: in the first case, $E_{\mathrm{DFG}}$ and $E_{\mathrm{SFG}}$ add up to a purely positive signal at the detector, as indicated by the green bar in Fig. 2a. Consequently, the measured photocurrent I is proportional to the square of the sum of both field vectors. This scenario may be implemented by detecting a band of frequencies in the center of the probe spectrum. Because the polarizations of $E_{\mathrm{DFG}}$ and $E_{\mathrm{SFG}}$ are perpendicular to $E_{\mathrm{p}}$, the latter may be suppressed by the linear polarizer. Because of the opposite rotation of $E_{\mathrm{DFG}}$ and $E_{\mathrm{SFG}}$, constructive and destructive interference occurs with varying time delays. Consequently, the resulting signal $S_{\text {center }}$ is proportional to the square of the MIR field, as sketched by the green graph in Fig. 2c. Note that phase information concerning $E_{\mathrm{THz}}$ is recovered in this way, although a local oscillator in form of the original probe is absent. 


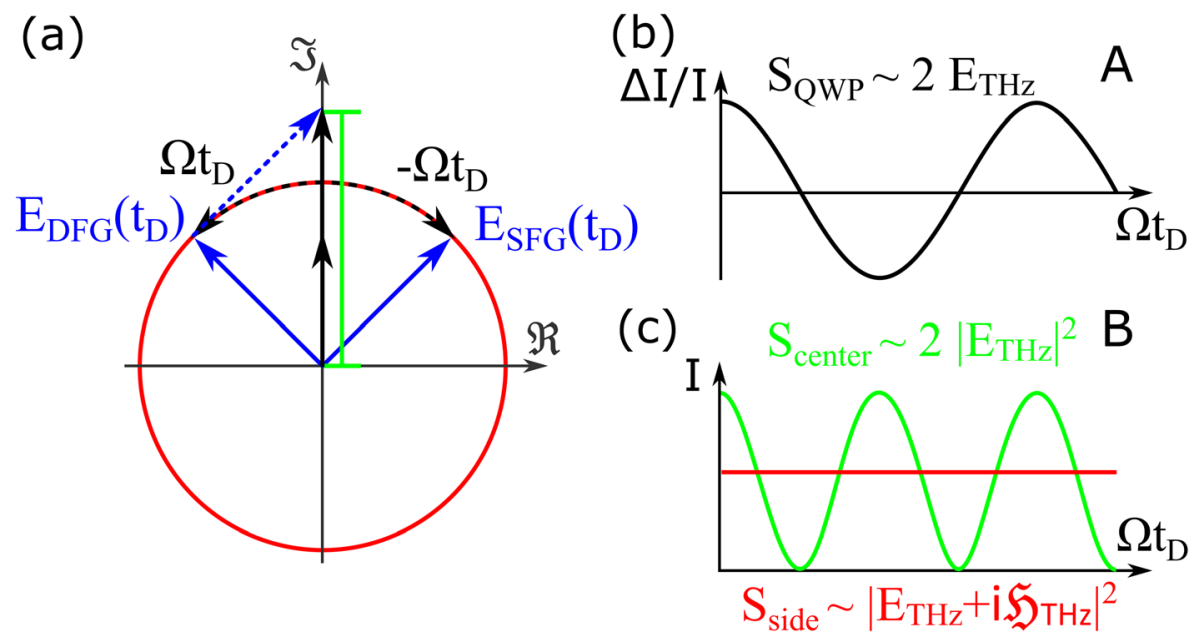

Fig. 2 (a) Phasor representation of electro-optic sampling in a complex plane. $\mathrm{E}_{\mathrm{SFG}}$ and $\mathrm{E}_{\mathrm{DFG}}$ are depicted as blue phasors at $t_{D} \neq 0$. The dotted black arrows illustrate the rotation direction of the new fields. The projection of the new fields to the imaginary axis $\mathfrak{I}$ is indicated by the black arrows. The blue-dotted phasor is inserted to illustrate the vector addition of the new fields. The absolute value of the result is indicated with a green bar. The red circle illustrates the constant absolute value of one of the fields, $\mathrm{E}_{\mathrm{SFG}}$ or $\mathrm{E}_{\mathrm{DFG}}$, respectively. (b) Differential signal $\Delta \mathrm{I} / \mathrm{I}$ versus time delay detected with scheme A. Signal $\mathrm{S}_{\mathrm{QWP}}$ presents the length of the black arrows and is proportional to $\mathrm{E}_{\mathrm{THz}}$. (c) Absolute current $\mathrm{I}$ of detection scheme $\mathrm{B}$ as a function of time delay. The green graph $\mathrm{S}_{\text {center }}$ illustrates the square of the green bar and the red signal $\mathrm{S}_{\text {side }}$ the square of the length of one single phasor $\mathrm{E}_{\mathrm{SFG}}$ or $\mathrm{E}_{\mathrm{DFG}}$

In the second case, either $E_{\mathrm{DFG}}$ or $E_{\mathrm{SFG}}$ is contributing to the signal exclusively. Experimentally, this goal may be implemented by detecting only photons on the high- or low-frequency sides of the probe spectrum, respectively. In this way, the suppression of any background due to $E_{\mathrm{p}}$ may be enhanced by an additional spectral filter which will turn out to be crucial for the experimental performance in Section 5. Owing to the absence of interference between $E_{\mathrm{DFG}}$ and $E_{\mathrm{SFG}}$, the output of this scheme becomes independent of $t_{\mathrm{D}}$, as illustrated with the red circle in the complex plane of Fig. 2a. From this simple consideration, we expect that the phase information vanishes completely in this scheme. This finding is somewhat surprising because in a static model of the electro-optic effect ("Pockels picture"), no signal should be generated at delay times where no electric field is applied to the EOX. Based on the frequency-domain picture of EOS and our phasor representation, an intuitive understanding of this context emerges from the fact that both conjugate variables, namely, the electric field $E_{\mathrm{THz}}$ and its Hilbert transform $\mathfrak{H}_{\mathrm{THz}}$, contribute equally to $E_{\mathrm{DFG}}$ and $E_{\mathrm{SFG}}$ and the signals are distinguished solely by their phase relationship with respect to $E_{\mathrm{p}}$. Consequently, we expect the total photocurrent I to be proportional to the intensity envelope of the MIR field which is defined as $\left|E_{\mathrm{THz}}+\mathrm{i} \mathfrak{H}_{\mathrm{THz}}\right|^{2}$. Therefore, the electric field and its conjugate variable remain indistinguishable.

\section{Numerical Calculations}

In the quasi-monochromatic phasor picture discussed in the previous section, two limiting cases were found where a background-free signal can either be proportional to the square or to the intensity envelope of the electric input field. In this section, a realistic scenario with 
broadband spectra is treated by a simulation of the intensity of the newly generated field $\left|E^{(2)}\right|^{2}$ $=\left|E_{\mathrm{DFG}}+E_{\mathrm{SFG}}\right|^{2}$. From the frequency-resolved numerical data, we can directly extract signals which are accessible with the background-free detection scheme B. In Fig. 3a, the calculated intensity $\left|E^{(2)}\right|^{2}$ of the new field is color-coded versus the frequency of the probe spectrum on the ordinate and the delay time $t_{\mathrm{D}}$ on the abscissa, respectively. For the simulation, the spectral shape of the input electric fields $E_{\mathrm{THz}}$ and $E_{\mathrm{p}}$ are chosen close to the experiment, but any phase dispersion is neglected. The MIR field is adopted as a Gaussian centered around a frequency of $35 \mathrm{THz}$ with a full width at half maximum (FWHM) of $16.65 \mathrm{THz}$. The normalized intensity of the probe spectrum centered at $280 \mathrm{THz}$ is superimposed to the left part of the color map (white graph with shading in Fig. 3a). It has a flat-top shape with inclined edges and exhibits a FWHM of $130 \mathrm{THz}$.

In Fig. 3b, the square $\left|E_{\mathrm{THz}}\right|^{2}$ and the intensity envelope $\left|E_{\mathrm{THz}}+\mathrm{i} \mathfrak{H}_{\mathrm{THz}}\right|^{2}$ of the multi-THz input field are plotted as a function of time delay $t_{\mathrm{D}}$ as a solid and a dashed black line, respectively. In addition, the newly generated NIR field intensities $\left|E^{(2)}\right|^{2}$ integrated over three spectral windows of a bandwidth of $10 \mathrm{THz}$ and centered at $210 \mathrm{THz}, 280 \mathrm{THz}$, and $350 \mathrm{THz}$ are depicted as magenta, green, and cyan graphs, respectively. These frequency intervals are also indicated in Fig. 3a with the same color coding.

The band pass (BP) filter at $280 \mathrm{THz}$ (green) is located in the center of the probe spectrum. The photons detected in this frequency band are generated equally by DFG and SFG. Note that the frequency distance between both boundaries of this filter interval and the upper and lower

Fig. 3 (a) Simulation of the intensity of the newly generated field $\left|E^{(2)}\right|^{2}=\left|E_{\mathrm{DFG}}+E_{\mathrm{SFG}}\right|^{2}$ colorcoded versus probe frequency and time delay. The normalized intensity spectrum of the probe pulse is plotted at left (white line with shading). The transmission ranges of three band pass (BP) filters are marked in magenta, green, and cyan. (b) The square $\left|E_{\mathrm{THz}}\right|^{2}$ of the $\mathrm{THz}$ input field and its intensity envelope $\left|E_{\mathrm{THz}}+i \mathfrak{H}_{\mathrm{THz}}\right|^{2}$ are plotted versus time delay as dashed and solid black lines, respectively. The intensities of the newly generated field $\left|E^{(2)}\right|^{2}$ integrated over the spectral slices indicated in (a) are depicted with the same color coding, respectively

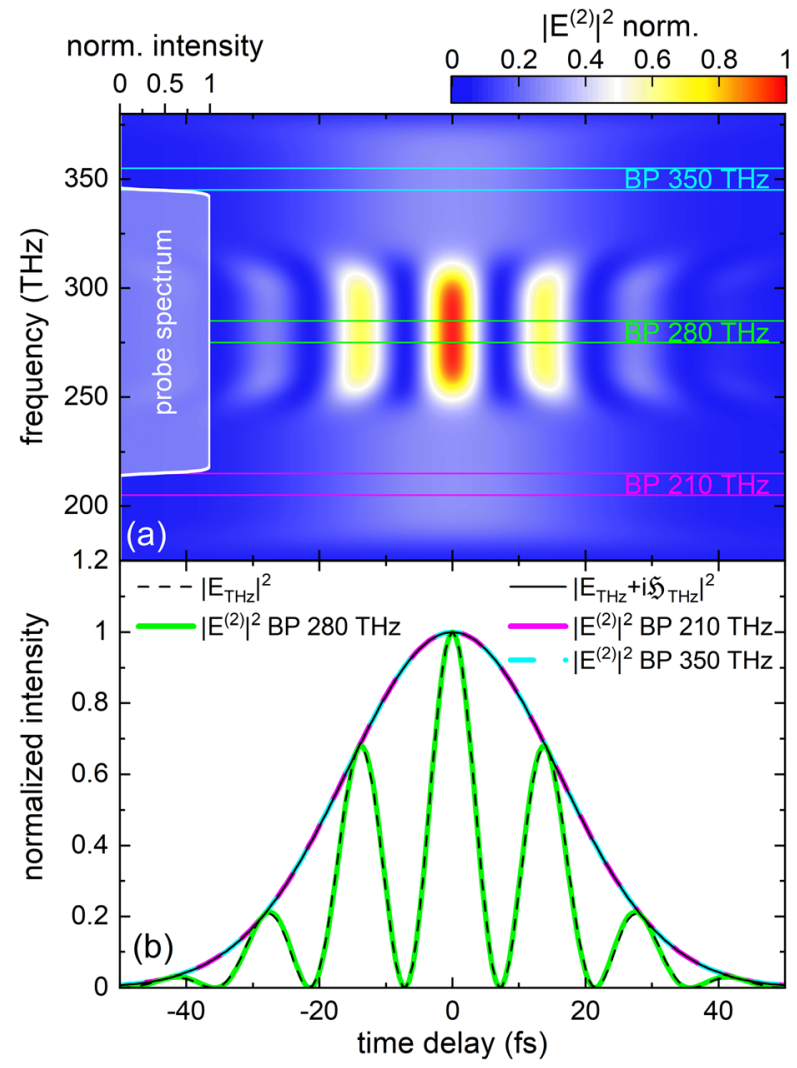


edge of the probe spectrum is as large as $60 \mathrm{THz}$, respectively. In this way, it is ensured that by far the dominant part of the frequency content of the THz input is able to generate both SFG and DFG falling into this frequency window. Therefore, the resulting signal (green graph in Fig. 3b) almost perfectly follows the square of the multi-THz transient $\left|E_{\mathrm{THz}}\right|^{2}$ (dashed black line).

For the second case, we consider the bandpass filters located close to the higher- (cyan) and lower-frequency (magenta) edges of the probe spectrum. This filter selection determines that also mixing products $\left|E^{(2)}\right|^{2}$ generated via SFG or DFG with the lower THz input frequencies may be detected, respectively. On the other side, the relatively narrow bandwidth of both filters in combination with the flat-top character of the probe ensures that MIR frequencies up to 130 $\mathrm{THz}$ may be detected with equal sensitivity. As expected from the qualitative argumentation in Section 3, both the signal emerging due to DFG around $210 \mathrm{THz}$ (magenta graph in Fig. 3b) and due to SFG around $350 \mathrm{THz}$ (cyan dashed line) are closely following the intensity envelope of the $\mathrm{THz}$ input (full black line).

\section{Experiments}

We now discuss experiments investigating the second case where either DFG or SFG photons are detected with scheme B (see Figs. 1 and 2). First, an edge filter (EF) with a cut-off frequency of $230 \mathrm{THz}$ is located before the detection crystal (EOX) to create a steep edge on the lower-frequency side of the probe spectrum. The normalized spectral intensity of the probe pulse is depicted as a green graph in Fig. 4a. A band pass (BP) filter centered at $222 \mathrm{THz}$ defines a detection window with a bandwidth of $8 \mathrm{THz}$ (red graph). A gap between the detection window and probe spectrum smaller than $10 \mathrm{THz}$ results. The linear polarizer additionally suppresses any non-interacting probe photons leaking into the detection window. The black graph with gray shading in Fig. 4a represents the intensity spectrum of the EOS transient $\mathrm{E}_{\mathrm{THz}}$ recorded with detection scheme A (black graph in Fig. 4b). The two black arrows illustrate examples of multi- $\mathrm{THz}$ frequencies falling into the detection window after DFG of probe frequencies with low- and high-frequency components of the MIR spectrum, respectively. The field envelope of the THz transient as calculated by $\left|E_{\mathrm{THz}}+\mathrm{i} \mathfrak{H}_{\mathrm{THz}}\right|$ is depicted in magenta. The photons within the detection window are recorded with an InGaAs photodiode (InGaAs PD). The resulting background-free signal is plotted in red as normalized current versus the time delay in Fig. 4c. The signal shows no modulation referring to MIR frequencies and thus proves the predictions of the previous sections that any phase information of the sampled field vanishes. In Fig. 4c, we now compare this signal with the intensity envelope $\left|E_{\mathrm{THz}}+\mathrm{i} \mathfrak{H}_{\mathrm{THz}}\right|^{2}$ of the EOS (dark green graph). The maxima of both time traces coincide in time, and their FWHM is quite similar with 25.8 fs for the intensity envelope and 28.5 fs for the background-free signal, respectively. These facts indicate that all frequencies of the nonlinear mixing products of the probe pulse and MIR transient are equally represented in the narrow detection window.

In a second measurement, the setup is configured to detect sum-frequency photons beyond the sharp high edge of the probe spectrum which has been shaped by the razor blade in the prism sequence (Fig. 5). The detection window is defined by a BP at $340 \mathrm{THz}$ with a bandwidth of $28 \mathrm{THz}$. In Fig. 5a, the resulting probe spectrum and the frequency band of the BP are depicted as normalized intensity and transmission in green and blue, respectively. Compared to the first setting, a larger gap is implemented between detection window and 
Fig. 4 Background-free detection of difference-frequency photons: (a) normalized intensity of probe spectrum (green) and BP transmission (red) versus frequency. The black graph represents the Fourier spectrum of the multi-THz transient shown as a black graph in (b) together with its field envelope $\left|E_{\mathrm{THz}}+\mathrm{i} \mathfrak{H}_{\mathrm{THz}}\right|$ (magenta line). (c) Normalized intensity envelope $\left|E_{\mathrm{THz}}+\mathrm{i}_{\mathrm{THz}}\right|^{2}$ (dark green) and photocurrent from the backgroundfree detection schema B (red) versus time delay

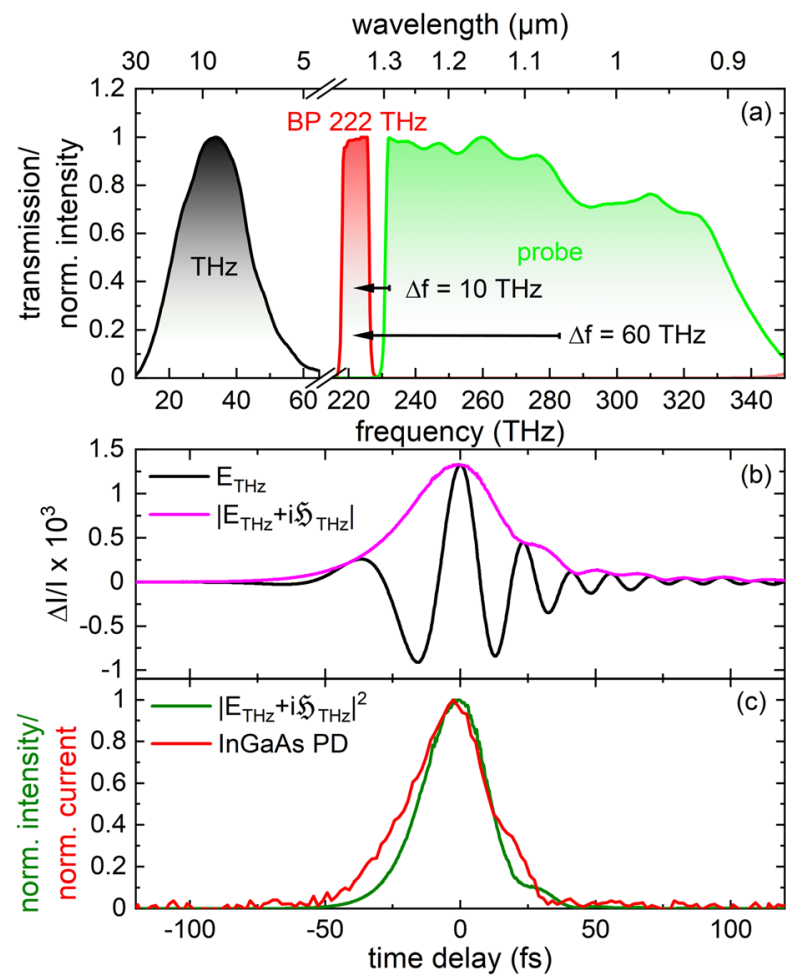

probe spectrum to investigate its influence on the MIR bandwidth detected in branch B. The black arrow illustrates the fact that the probe photons have to mix with MIR frequencies via $\mathrm{SFG}$ at least as high as $30 \mathrm{THz}$ to reach the detection window. The multi-THz transient $E_{\mathrm{THz}}$ is analyzed with the modified probe spectrum by EOS and plotted in black as a function of time delay in Fig. 5b. Again, the calculated field envelope is depicted in magenta. In Fig. 5c, the resulting intensity envelope of the multi- $\mathrm{THz}$ transient $\left|E_{\mathrm{THz}}+i \mathfrak{H}_{\mathrm{THz}}\right|^{2}$ is plotted in dark green together with the normalized photocurrent detected by a Si-APD in branch B (blue graph). As for the DFG process, a modulation-free signal also results in this case, and the temporal positions of the maxima of both signals are located at the same time delay. This time, however, the background signal is significantly broadened. This fact results directly from the increased spectral gap between the probe frequencies and the detection window. In this configuration, the new photons generated by SFG with MIR frequencies below $30 \mathrm{THz}$ cannot contribute to the background-free signal. Thus, the detectable bandwidth of the MIR spectrum is limited to higher frequency components, and we observe a wave packet which is stretched in time.

We now discuss an option for background-free detection where some information on the phase of the THz input field is to be recovered. As discussed in Section 4, the detection window then has to be located in the center of probe spectrum where DFG and SFG contribute equally. Therefore, we measure with a BP centered at $272 \mathrm{THz}$. In this case, clean polarization states become even more important because the suppression of the probe relies on the linear polarizer only. In our setup, the purity of the polarization is strongly influenced by the tight focus into the EOX and subsequent recollimation. The extinction ratio decreases to $1: 10^{3}$ from the original ratio of $1: 10^{6}$ determined before the 
Fig. 5 Background-free detection of sum-frequency photons: (a) normalized intensity of probe spectrum (green) and BP transmission (blue) versus frequency. (b) EOS signal of the multi-THz transient in black with its field envelope $\left|E_{\mathrm{THz}}+\mathrm{i}_{\mathrm{THz}}\right|$ (magenta).

(c) Normalized intensity envelope $\left|E_{\mathrm{THz}}+\mathrm{i} \mathfrak{H}_{\mathrm{THz}}\right|^{2}$ (dark green) and photocurrent from the backgroundfree detection schema B (blue) versus time delay
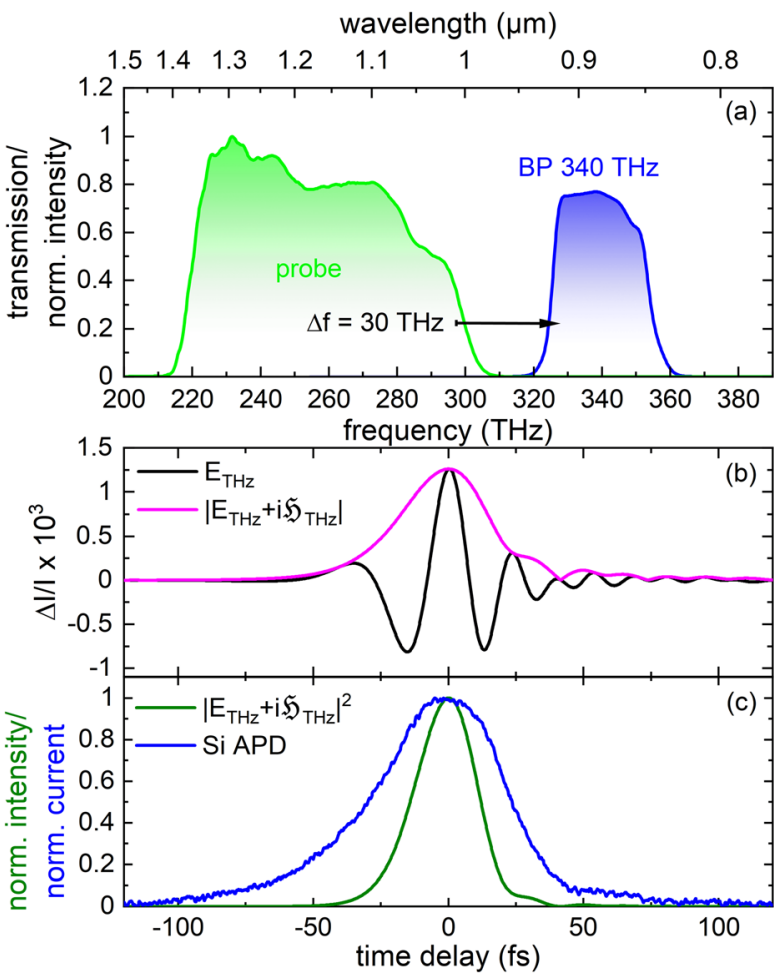

focusing off-axis paraboloid. Therefore, the original probe photons cannot be suppressed completely and an offset remains. The signal induced by mixing with the MIR pulse train is extracted by modulating it with half the repetition rate of the pulse train and detection with a lock-in amplifier. The resulting signal is plotted in green in Fig. 6a. The negative parts of this transient emerge from destructive interference between the newly generated NIR photons and the spurious background due to the imperfect polarization filtering of the probe which is subtracted by the lock-in. For comparison, a second ordinate on the right shows the absolute value of the photocurrent including the background which exhibits purely positive values. The square of the $\mathrm{THz}$ transient $\left|\mathrm{E}_{\mathrm{THz}}\right|^{2}$ shown in black is calculated from the EOS trace. Pronounced differences in amplitude and phase between the measured (green) and the expected (black) results are evident. The measured signal is neither proportional to the multi- THz transient itself nor to its square. This finding originates from the leaking probe photons which partly interfere with the mixed contributions of the SFG and DFG components. This result may be understood by a projection of the field amplitudes to a polarizer with slightly misaligned transmission axis. By adding up the contributions of $E_{\mathrm{p}}$ and $E^{(2)}$ in analogy to the calculations in Ref. [9], one finds terms which are linear and quadratic in $E_{\mathrm{THz}}$.

To explain this result in an intuitive way, we consider the conventional picture which is based on the nonlinear phase shift induced by $E_{\mathrm{THz}}$ in the EOX $[19,20]$. This limit represents a good approximation in the present scenario where DFG and SFG are equally contributing to the signal. The photocurrent $I_{\mathrm{S}}$ resulting for a linear polarizer in direction $\mathrm{e}_{\mathrm{s}}$ is plotted as a function of the nonlinear phase shift $\Gamma$ in Fig. $6 b$ where 


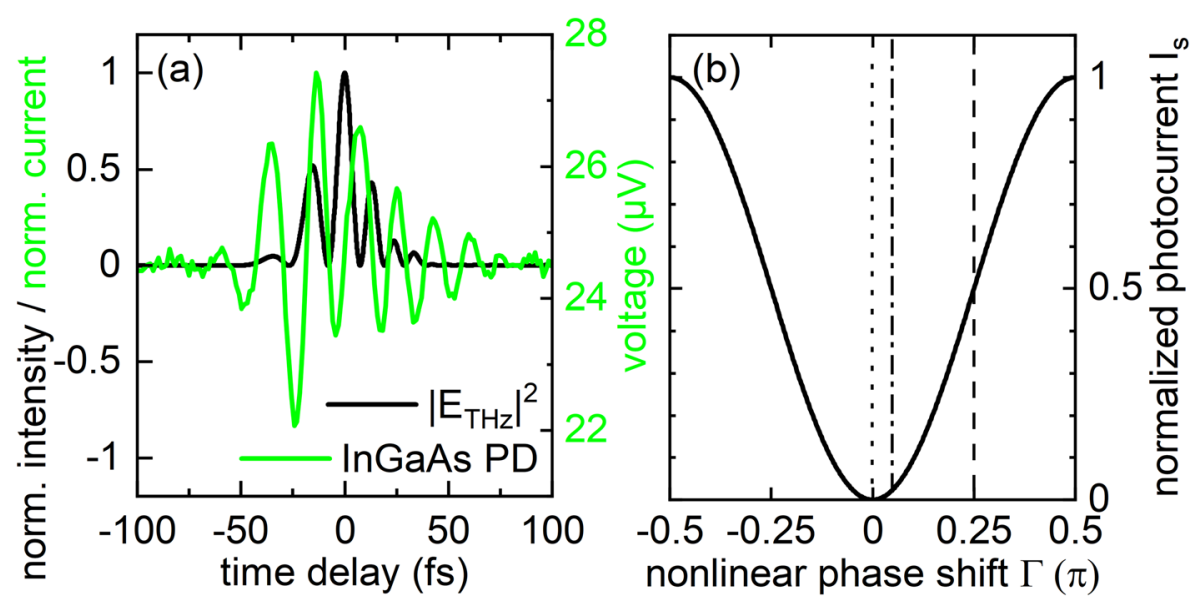

Fig. 6 (a) Detection of sum- and difference-frequency photons in the center of probe spectrum with a BP at 272 THz. A normalized current output from detection scheme B is plotted in green versus time delay. The right ordinate shows the absolute voltage of the measurement including the offset due to leaking probe photons. The black graph represents the square of the results obtained with scheme A for the same input signal. (b) Normalized photocurrent calculated theoretically for a polarizer in direction $\mathrm{e}_{\mathrm{s}}$ and no spectral filtering applied as a function of the nonlinear phase shift $\Gamma$ which is proportional to the MIR transient $\mathrm{E}_{\mathrm{THz}}$. The ideal setpoint for this configuration is indicated by the vertical dotted line. A linear regime is reached by optical biasing with a quarter-wave plate in the standard configuration of EOS, as indicated with the dashed vertical line. In a realistic experiment, a background signal emerges due to the finite leakage of the probe electric field which is nominally polarized along the $e_{z}$ direction. Such a situation is indicated by the dash-dotted vertical line

$$
I_{s}(\Gamma)=I_{p} \sin ^{2}(\Gamma) .
$$

$I_{\mathrm{p}}$ is the intensity of the original probe parallel to the direction of $\mathrm{e}_{\mathrm{z}}$. The phase shift $\Gamma=\Gamma_{0}+$ $\Gamma_{\mathrm{THz}}$ consists of two parts. A static offset $\Gamma_{0}$ and an induced nonlinear phase shift $\Gamma_{\mathrm{THz}}$, which is proportional to the change of the refractive index $\Delta n$ emerging from the quasi-static electric field $\mathrm{E}_{\mathrm{THz}}$ via the Pockels effect:

$$
\Gamma_{\mathrm{THz}}=\frac{\omega \Delta n l}{c}=\frac{\omega r_{41} n_{0}^{3} l}{2 c} E_{T H z},
$$

with the center frequency of the probe $\omega$, the electro-optic coefficient $r_{41}$, the linear refractive index $n_{0}$, and the thickness of the EOX $l$.

First, we discuss the standard situation for electro-optic sampling. Here, a QWP adds an optical bias of $\Gamma_{0}=0.25 \pi$. This biasing point is indicated with a vertical dashed line in Fig. $6 \mathrm{~b}$. For small phase shifts $\Gamma_{\mathrm{THz}}$, the signal $I_{\mathrm{s}}$ varies linearly with the multi-THz field amplitude $E_{\mathrm{THz}}$. In contrast, the signal $I_{s}$ is proportional to $\left|E_{\mathrm{THz}}\right|^{2}$ for $\Gamma_{0}=0$ (dotted vertical line in Fig. 6b). The dash-dotted line indicates a realistic setpoint with a small background signal creating a finite offset $\Gamma_{0}>0$. In this picture, it is straightforward to see that the ratio of the offset $\Gamma_{0}$ compared to the induced nonlinear phase-shift $\Gamma_{\mathrm{THz}}$ is the decisive factor for detecting a signal which is either linear in $E_{\mathrm{THz}}$ or proportional to $\left|E_{\mathrm{THz}}\right|^{2}$ : when $\Gamma_{\mathrm{THz}}$ is large owing to a strong $\mathrm{THz}$ field and thick EOX [11], the static offset $\Gamma_{0}$ becomes negligible and a signal reflecting $\left|E_{\mathrm{THz}}\right|^{2}$ results. Instead, we expect a change of $I_{\mathrm{s}}$ which is proportional to $E_{\mathrm{THz}}$ as long as $\Gamma_{\mathrm{THz}}<<\Gamma_{0}$. The green data set in Fig. 6a obviously represents a transitional situation between both scenarios. 
This situation near the so-called zero transition point is related to detection schemes presented in several works [12, 21-23]. Here, an increase in the signal-to-noise ratio is achieved by boosting the number of probe photons in the EOX while maintaining constant optical power on the balanced photodetector. This fact avoids saturation effects in the photodiodes. The shot noise of the probe remains as the limiting factor for the sensitivity, however.

\section{Summary}

Motivated by pushing the sensitivity limits for time-domain sampling of electric field amplitudes at infrared and optical frequencies, we have explored options which are not limited by the shot noise introduced due to homodyning with the subcycle readout pulses. Circumventing this limitation originating from the quantum nature of the gate pulses will enable more thorough examination of the subcycle quantum properties of the MIR pulses themselves. A first scheme relies on detection of photons generated by either sum- or difference-frequency mixing in a spectral range outside the bandwidth of the ultrashort probe. Here, the surprising insight is represented by the fact that interaction of the terahertz input field or its Hilbert transform result in exactly the same mixing efficiency with the subcycle probe. In this extreme limit of nonlinear optics, the phase modulation due to the temporal gradient present in zero crossings of the terahertz field obviously generates the same output amplitude as the phase shift due to the electro-optic effect in the field extrema. This statement holds independently of the carrier frequency of the input, further underlining the role of the Hilbert transform as a conjugate variable to the electric field in the time domain. The only difference occurs in the phase of the mixing product which is accessible only with some kind of interference effect. In the absence of the latter, only amplitude information is recovered, and all phase information is lost. This behavior is changed when allowing for interference between components generated by sum- and difference-frequency mixing. In this case, the background due to original probe photons may still be suppressed by polarization filtering, and a signal proportional to the square of the electric input field is expected. Nevertheless, it turns out that any spurious leakage of the probe field still leads to a signal component linear in the input field which is limited by shot noise. This component gains importance especially when working with low input field amplitudes which are expected in any time-domain quantum technology.

Code Availability Not applicable

Funding Open Access funding enabled and organized by Projekt DEAL. This work is funded by the Deutsche Forschungsgemeinschaft (DFG) - Project-ID 425217212 - SFB 1432.

Data availability Not applicable

\section{Declarations}

Conflict of Interest The authors declare no competing interests. 
Open Access This article is licensed under a Creative Commons Attribution 4.0 International License, which permits use, sharing, adaptation, distribution and reproduction in any medium or format, as long as you give appropriate credit to the original author(s) and the source, provide a link to the Creative Commons licence, and indicate if changes were made. The images or other third party material in this article are included in the article's Creative Commons licence, unless indicated otherwise in a credit line to the material. If material is not included in the article's Creative Commons licence and your intended use is not permitted by statutory regulation or exceeds the permitted use, you will need to obtain permission directly from the copyright holder. To view a copy of this licence, visit http://creativecommons.org/licenses/by/4.0/.

\section{References}

1. C. Riek et al., "Direct sampling of electric-field vacuum fluctuations," Science (80-. )., vol. 350, no. 6259, pp. 420-423, Oct. 2015, doi: https://doi.org/10.1126/science.aac9788.

2. I. C. Benea-Chelmus, F. F. Settembrini, G. Scalari, and J. Faist, "Electric field correlation measurements on the electromagnetic vacuum state," Nature, vol. 568, no. 7751, pp. 202-206, 2019, doi: https://doi.org/10. 1038/s41586-019-1083-9.

3. C. Riek, "Subcycle quantum electrodynamics," Nat. Publ. Gr., vol. 541, no. 7637, pp. 376-379, 2017, doi: https://doi.org/10.1038/nature21024.

4. M. Kizmann, T. L. de M. Guedes, D. V. Seletskiy, A. S. Moskalenko, A. Leitenstorfer, and G. Burkard, "Subcycle squeezing of light from a time flow perspective," 2018, [Online]. Available: http://arxiv.org/abs/ 1807.10519.

5. T. L. M. Guedes, M. Kizmann, D. V. Seletskiy, A. Leitenstorfer, G. Burkard, and A. S. Moskalenko, "Spectra of Ultrabroadband Squeezed Pulses and the Finite-Time Unruh-Davies Effect," Phys. Rev. Lett., vol. 122, no. 5, p. 53604, 2019, doi: https://doi.org/10.1103/PhysRevLett.122.053604.

6. Q. Wu and X. C. Zhang, "Free-space electro-optic sampling of terahertz beams," Appl. Phys. Lett., vol. 67, no. September 1995, p. 3523, 1995, doi: https://doi.org/10.1063/1.114909.

7. C. Kübler, R. Huber, and A. Leitenstorfer, "Ultrabroadband terahertz pulses: Generation and field-resolved detection," Semicond. Sci. Technol., vol. 20, no. 7, 2005, doi: https://doi.org/10.1088/0268-1242/20/7/002.

8. G. Gallot and D. Grischkowsky, "Electro-optic detection of terahertz radiation," J. Opt. Soc. Am. B, vol. 16, no. 8, p. 1204, 1999, doi: https://doi.org/10.1364/josab.16.001204.

9. P. Sulzer et al., "Determination of the electric field and its Hilbert transform in femtosecond electro-optic sampling," vol. 033821, pp. 1-17, 2020, doi: https://doi.org/10.1103/PhysRevA.101.033821.

10. J. van Tilborg, D. J. Bakker, N. H. Matlis, and W. P. Leemans, "Spectral sidebands on a narrow-bandwidth optical probe as a broad-bandwidth THz pulse diagnostic," Opt. Express, vol. 19, no. 27, p. 26634, 2011, doi: https://doi.org/10.1364/oe.19.026634.

11. D. A. Walsh, E. W. Snedden, and S. P. Jamison, "The time resolved measurement of ultrashort terahertzband electric fields without an ultrashort probe," Appl. Phys. Lett., vol. 106, no. 18, 2015, doi: https://doi. org/10.1063/1.4919899.

12. J. A. Johnson, F. D. J. Brunner, S. Grübel, A. Ferrer, S. L. Johnson, and T. Feurer, "Distortion-free enhancement of terahertz signals measured by electro-optic sampling II Experiment," J. Opt. Soc. Am. B, vol. 31, no. 5, p. 1035, 2014, doi: https://doi.org/10.1364/josab.31.001035.

13. M. Cornet, J. Degert, E. Abraham, and E. Freysz, "Frequency resolved cross-correlation between optical and terahertz pulses: application to ultrashort laser pulse characterization," Opt. Express, vol. 24, no. 3, p. 3003, 2016, doi: https://doi.org/10.1364/OE.24.003003.

14. E. W. Snedden, D. A. Walsh, and S. P. Jamison, "Revealing carrier-envelope phase through frequency mixing and interference in frequency resolved optical gating," Opt. Express, vol. 23, no. 7, p. 8507, 2015, doi: https://doi.org/10.1364/oe.23.008507.

15. M. Clerici et al., "Counter-propagating difference frequency mixing in diamond with terahertz waves," 2013 Conf. Lasers Electro-Optics, CLEO 2013, vol. 38, no. 2, pp. 178-180, 2013, doi: https://doi.org/10. 1364/cleo_si.2013.cm4j.5.

16. M. Clerici et al., "Spectrally resolved wave-mixing between near- and far-infrared pulses in gas," New J. Phys., vol. 15, 2013, doi: https://doi.org/10.1088/1367-2630/15/12/125011.

17. M. Wunram, P. Storz, D. Brida, and A. Leitenstorfer, "Ultrastable fiber amplifier delivering 145 -fs pulses with 6- $\mu \mathrm{J}$ energy at 10-MHz repetition rate," Opt. Lett., vol. 40, no. 5, p. 823, 2015, doi: https://doi.org/10. 1364/ol.40.000823. 
18. P. Storz, J. Tauch, M. Wunram, A. Leitenstorfer, and D. Brida, "Parametric Amplification of Phase-Locked Few-Cycle Pulses and Ultraviolet Harmonics Generation in Solids at High Repetition Rate," Laser Photonics Rev., vol. 11, no. 6, pp. 1-5, 2017, doi: https://doi.org/10.1002/lpor.201700062.

19. C. Riek, D. V. Seletskiy, and A. Leitenstorfer, "Femtosecond measurements of electric fields: From classical amplitudes to quantum fluctuations," Eur. J. Phys., vol. 38, no. 2, pp. 1-13, 2017, doi: https://doi.org/10. 1088/1361-6404/aa53a2.

20. Q. Chen, M. Tani, Z. Jiang, and X.-C. Zhang, "Electro-optic transceivers for terahertz-wave applications," J. Opt. Soc. Am. B, vol. 18, no. 6, p. 823, 2001, doi: https://doi.org/10.1364/josab.18.000823.

21. Z. Jiang, F. G. Sun, Q. Chen, and X. C. Zhang, "Electro-optic sampling near zero optical transmission point, " Appl. Phys. Lett., vol. 74, no. 9, pp. 1191-1193, 1999, doi: https://doi.org/10.1063/1.123495.

22. J. Degert, M. Cornet, E. Abraham, and E. Freysz, "Simple and distortion-free optical sampling of terahertz pulses via heterodyne detection schemes," J. Opt. Soc. Am. B, vol. 33, no. 10, p. 2045, 2016, doi: https://doi. org/10.1364/josab.33.002045.

23. P. Krauspe, N. Banerji, and J. Réhault, "Effective detection of weak terahertz pulses in electro-optic sampling at kilohertz repetition rate," J. Opt. Soc. Am. B, vol. 37, no. 1, p. 127, 2020, doi: https://doi.org/ 10.1364/josab.37.000127.

Publisher's Note Springer Nature remains neutral with regard to jurisdictional claims in published maps and institutional affiliations. 